\title{
Generating M-indeterminate probability densities by way of quantum mechanics
}

\author{
Rafael Sala Mayato ${ }^{a}$, Patrick Loughlin ${ }^{b}$, Leon Cohen $^{c *}$ \\ ${ }^{a}$ Departamento de Física and IUdEA, Universidad de La Laguna, La Laguna 38203, Tenerife, Spain \\ ${ }^{b}$ Departments of Bioengineering, and Electrical \& Computer Engineering, University of Pittsburgh, \\ Pittsburgh, PA 15261, USA \\ ${ }^{c}$ Department of Physics, Hunter College of the City University of New York, 695 Park Ave., New \\ York, NY 10065, USA. *Corresponding author; email: leon.cohen@hunter.cuny.edu
}

\begin{abstract}
Probability densities that are not uniquely determined by their moments are said to be "moment-indeterminate," or "M-indeterminate." Determining whether or not a density is Mindeterminate, or how to generate an M-indeterminate density, is a challenging problem with a long history. Quantum mechanics is inherently probabilistic, yet the way in which probability densities are obtained is dramatically different in comparison to standard probability theory, involving complex wave functions and operators, among other aspects. Nevertheless, the end results are standard probabilistic quantities, such as expectation values, moments and probability density functions. We show that the quantum mechanics procedure to obtain densities leads to a simple method to generate an infinite number of M-indeterminate densities. Different self-adjoint operators can lead to new classes of M-indeterminate densities. Depending on the operator, the method can produce densities that are of the Stieltjes class or new formulations that are not of the Stieltjes class. As such, the method complements and extends existing approaches and opens up new avenues for further development. The method applies to continuous and discrete probability densities. A number of examples are given.
\end{abstract}

Keywords: M-indeterminate probability densities; moments; quantum mechanics; Stieltjes class

\section{Introduction}

Let $X$ be a continuous real random variable with associated probability density $P(x)\left(x \in \mathbb{R}^{1}\right)$ and moments $\mathrm{E}\left[X^{n}\right]$, where $n$ is a positive integer and $\mathrm{E}[$.$] denotes the expected value of the argument.$ Many of the common densities in statistics are uniquely determined by their moments; however, some are not. Probability densities that are not uniquely determined by their moments are said to be "moment-indeterminate," or "M-indeterminate." The idea first arose with an example by Stieltjes but the best known one is the log-normal density [1, 2],

$$
P_{\mathrm{LN}}(x)=\frac{1}{x \sqrt{2 \pi}} \exp \left[-\frac{(\ln x)^{2}}{2}\right] \quad 0<x<\infty
$$

which has moments

$$
\mathrm{E}\left[X^{n}\right]=\int_{0}^{\infty} x^{n} P_{\mathrm{LN}}(x) d x=e^{n^{2} / 2}
$$

In a landmark paper, Heyde [3] explicitly showed that the densities given by

$$
P(x)=P_{\mathrm{LN}}(x)[1+\varepsilon \sin (k 2 \pi \ln x)], \quad-1 \leq \varepsilon \leq 1
$$


where $k \in \mathbb{I}^{+}$, have the same moments as $P_{\mathrm{LN}}(x)$. Thus, the log-normal is M-indeterminate. Determining whether or not a given density is M-indeterminate, or how to generate an M-indeterminate density, is a challenging problem with a long history in probability theory [2]-[1].

The "Stieltjes class" of M-indeterminate densities can be formulated as a parameterized family of functions [6, 7]

$$
P_{\epsilon}(x)=P(x)[1+\varepsilon h(x)], \quad-1 \leq \varepsilon \leq 1
$$

where $P(x)$ is an M-indeterminate density, and $h(x) \neq 0$ is a continuous, bounded $(|h(x)| \leq 1)$ function called a "perturbation function," subject to the condition that the product $h(x) P(x)$ has vanishing "moments,"

$$
\int_{-\infty}^{\infty} x^{n} h(x) P(x) d x=0, \quad n=0,1,2, \ldots
$$

Stoyanov and Tolmatz have developed a powerful method for constructing Stieltjes classes of Mindeterminate densities [8]. Briefly, the method consists of specifying the scale $\delta$ and shift $s$ parameters for some continuous, bounded function $g(x)$ with vanishing moments, such that

$$
h(x)=c \frac{g(\delta x-s)}{P(x)}
$$

exists for all $x$, where $c$ is a normalization constant. There are several candidate functions $g(x)$, and convex combinations of such functions can also be used [8]. A number of examples of M-indeterminate densities, and detailed discussion of the moment problem, can be found in the book by Stoyanov [2].

We provide a different perspective on the problem of generating M-indeterminate densities that derives from the way in which probability densities are obtained in quantum mechanics (QM). While QM is inherently probabilistic, the mathematics underlying it are quite different in comparison to standard probability theory, involving complex wave functions, operators, transformation theory and other aspects. We show how one can generate different classes of M-indeterminate densities by choosing different self-adjoint operators.

Operators are central in QM and correspond to measurable quantities or "observables," such as energy, position, momentum, etc. Operator methods have also been used in standard probability theory, mostly involving the differentiation operator, which arises in the study of cumulants [12, 13. and the Gram-Charlier series [14]. For a comparison of the quantum and standard approach to obtaining probabilities, see [15, 16].

Despite the unorthodox methods of QM, we emphasize that the densities obtained are proper in the classical sense. Moreover, the mathematics of QM bring forth new ideas and approaches that can inform standard probability theory. The aim of this paper is to show that the procedure by which densities are obtained in QM leads to a simple procedure for generating new classes of M-indeterminate densities, that complements existing approaches and opens up new avenues for further development.

\section{Preliminaries: Notation, densities and expectation values in QM}

To set forth our notation and because of its distinction from standard probability theory, we briefly review how probability densities are obtained in QM [17, 18].

Measureable physical quantities, such as the position or momentum of a particle, are inherently probabilistic and associated with self-adjoint operators in QM. The measurable quantities are obtained 
from the quantum mechanical wave function, often denoted by $\psi(x)$, which is complex and represents the full description of the physical system.

Definition 1 For a continuous random variable $X$ and wave function $\psi(x)\left(x \in \mathbb{R}^{1}\right)$ in $L^{2}$, the QM probability density associated with $\psi(x)$ is

$$
P(x)=|\psi(x)|^{2}
$$

with normalization

$$
\int_{-\infty}^{\infty} P(x) d x=\int_{-\infty}^{\infty}|\psi(x)|^{2} d x=1
$$

The unique aspect of QM is how densities of other variables are obtained, which is done by solving an eigenvalue problem,

$$
\mathbf{A} u(r, x)=r u(r, x)
$$

where $\mathbf{A}$ is a self-adjoint operator that represents a physical quantity, and the $r$ 's are the eigenvalues, which are the random variables in QM. The $u$ 's are the corresponding eigenfunctions, which are complete and orthogonal. Accordingly, $\psi(x)$ as defined may be expanded as [19]

$$
\psi(x)=\int_{-\infty}^{\infty} F(r) u(r, x) d r
$$

where

$$
F(r)=\int_{-\infty}^{\infty} \psi(x) u^{*}(r, x) d x
$$

The function $F(r)$ is the representation of the function in the $r$-domain, and we shall call it the $r$ transform of $\psi(x)$. In writing Eq. (3), we have assumed the variables are continuous; the discrete case is considered in sec. 5 ,

Definition 2 For a continuous random variable $R$ and transform $F(r)\left(r \in \mathbb{R}^{1}\right)$ in $L^{2}$ as defined in Eq. (4), the QM probability density associated with $F(r)$ is

$$
P(r)=|F(r)|^{2}=\left|\int_{-\infty}^{\infty} \psi(x) u^{*}(r, x) d x\right|^{2}
$$

Note that $|F(r)|^{2}$ is a proper density, in that it is non-negative, and properly normalized since $|\psi(x)|^{2}$ was normalized,

$$
\int_{-\infty}^{\infty}|F(r)|^{2} d r=\int_{-\infty}^{\infty}|\psi(x)|^{2} d x=1
$$

Lemma 1 Assume $R$ is a continuous random variable with density $|F(r)|^{2}\left(r \in \mathbb{R}^{1}\right)$ and $F(r)$ given by Eq. (4). Then the average value of $R$, defined by

$$
\mathrm{E}[R]=\int_{-\infty}^{\infty} r|F(r)|^{2} d r
$$


can be equivalently calculated in terms of $\psi(x)$ by way of

$$
\mathrm{E}[R]=\int_{-\infty}^{\infty} \psi^{*}(x) \mathbf{A} \psi(x) d x
$$

More generally, for a real function $g(R)$, its average value is given by

$$
\mathrm{E}[g(R)]=\int_{-\infty}^{\infty} g(r)|F(r)|^{2} d r=\int_{-\infty}^{\infty} \psi^{*}(x) g(\mathbf{A}) \psi(x) d x
$$

Proof. See [17, 18] and references therein.

\section{Generating M-indeterminate densities}

We now make use of the procedures by which densities and expectation values are obtained in QM to obtain new classes of M-indeterminate probability densities. In what follows, $R$ represents a continuous real random variable with associated probability density $P(r)$, and $\psi(x)$ is any complex function satisfying the conditions of Theorem 1.

Theorem 1 Let $\mathbf{A}$ be any self-adjoint operator with corresponding eigenfunctions $u(r, x)$, for which the support of $\mathbf{A}^{n} \psi(x)$ equals the support of $\psi(x), n \in \mathbb{I}^{+}$. Further, let $\psi_{1}(x) \neq 0$ and $\psi_{2}(x) \neq 0$ be any normalized generally complex functions in $L^{2}$ having disjoint supports $\left(\psi_{1}(x) \psi_{2}(x)=0\right)$. Then, for the family of functions

$$
\psi(x)=\frac{1}{\sqrt{2}}\left(\psi_{1}(x)+e^{i \beta} \psi_{2}(x)\right), \quad-\pi \leq \beta \leq \pi
$$

the classes of densities given by

$$
P(r)=\left|\int_{-\infty}^{\infty} \psi(x) u^{*}(r, x) d x\right|^{2}
$$

depend on the parameter $\beta$ but the moments $\mathrm{E}\left[R^{n}\right]$ are independent of $\beta$. Hence, the classes of densities $P(r)$ are $M$-indeterminate.

Proof. Substituting $\psi(x)$ from Eq. (8) into Eq. (9), we have

$$
P(r)=\left|\frac{1}{\sqrt{2}}\left(F_{1}(r)+e^{i \beta} F_{2}(r)\right)\right|^{2}=\frac{1}{2}\left(\left|F_{1}(r)\right|^{2}+\left|F_{2}(r)\right|^{2}+2 \operatorname{Re}\left\{e^{i \beta} F_{1}^{*}(r) F_{2}(r)\right\}\right)
$$

where

$$
F_{k}(r)=\int_{-\infty}^{\infty} \psi_{k}(x) u^{*}(r, x) d x, \quad k=1,2
$$

Clearly, the densities $P(r)$ depend on $\beta$. For the moments, we have

$$
\mathrm{E}\left[R^{n}\right]=\int_{-\infty}^{\infty} r^{n} P(r) d r=\frac{1}{2} \int_{-\infty}^{\infty} r^{n}\left(\left|F_{1}(r)\right|^{2}+\left|F_{2}(r)\right|^{2}+2 \operatorname{Re}\left\{e^{i \beta} F_{1}^{*}(r) F_{2}(r)\right\}\right) d r
$$


or equivalently, by Eq. (77),

$$
\begin{aligned}
\mathrm{E}\left[R^{n}\right] & =\int_{-\infty}^{\infty} \psi^{*}(x) \mathbf{A}^{n} \psi(x) d x \\
& =\frac{1}{2} \int_{-\infty}^{\infty} \psi_{1}^{*}(x) \mathbf{A}^{n} \psi_{1}(x)+\psi_{2}^{*}(x) \mathbf{A}^{n} \psi_{2}(x)+e^{i \beta} \psi_{1}^{*}(x) \mathbf{A}^{n} \psi_{2}(x)+e^{-i \beta} \psi_{2}^{*}(x) \mathbf{A}^{n} \psi_{1}(x) d x
\end{aligned}
$$

Hence, in order for the moments $\mathrm{E}\left[R^{n}\right]$ to be independent of $\beta$, we must have

$$
\int_{-\infty}^{\infty} e^{i \beta} \psi_{1}^{*}(x) \mathbf{A}^{n} \psi_{2}(x)+e^{-i \beta} \psi_{2}^{*}(x) \mathbf{A}^{n} \psi_{1}(x) d x=0
$$

This condition follows immediately by the fact that $\psi_{1}(x)$ and $\psi_{2}(x)$ have disjoint support, and the support of $\mathbf{A}^{n} \psi_{k}(x)$ equals the support of $\psi_{k}(x)$; therefore the integrands are zero.

Remark 1 This theorem generates various classes of M-indeterminate densities in three ways:

(a) First, for a given $\psi_{1}(x), \psi_{2}(x)$ and self adjoint operator $\mathbf{A}$, one has a particular family of densities, with different members defined by the value of the parameter $\beta$. This is analogous to the lognormal family as parameterized by $\varepsilon$ in Eq. (11), or the Stieltjes class of Eq. (2) for a specific $P(x)$.

(b) Second and more significantly, for a given $\psi_{1}(x)$ and $\psi_{2}(x)$, different self-adjoint operators yield different classes of M-indeterminate densities. Examples of different classes generated by choosing different self-adjoint operators are provided in the next section.

(c) Analogously, for a given self-adjoint operator, different classes are defined by choosing different non-overlapping functions $\psi_{1}(x)$ and $\psi_{2}(x)$.

One way to obtain such functions is to use "bump functions," which are compactly supported differentiable functions [24]; there is a variety of such functions, one example being the family of functions

$$
f(x)= \begin{cases}e^{-\frac{1}{1-x^{2 k}}} & |x|<1 \\ 0, & \text { otherwise }\end{cases}
$$

where $k$ is a positive integer. Hence, one can take $\psi_{1}(x)$ to be a unit-normalized bump function, e.g., $\psi_{1}(x)=c f(x)$ (where $c$ is a normalization constant), which equals zero for $|x|>1$. Then take $\psi_{2}(x)$ to be a shifted and possibly also scaled version of $\psi_{1}$, namely $\psi_{2}(x)=\sqrt{\alpha} \psi_{1}(\alpha(x \pm D))$ with $\alpha>0$ and $D>1+\frac{1}{\alpha}$, by which it follows that $\psi_{1}(x) \psi_{2}(x)=0$, as required.

Remark 2 The specific wave function given by Eq. (8) was considered in the QM context to show that the moments of position and momentum are independent of $\beta[20,21,22$. This result is known as one of Aharonov's paradoxes. The issue has been recently revisited in terms of the characteristic function approach to generating densities [23]. For our purposes here, $\psi(x)$ is any function satisfying the conditions of Theorem 1 . 


\section{Examples of Different Classes of Densities}

We now consider various self-adjoint operators and the corresponding M-indeterminate densities. As noted in Remark 1, each operator generates a new class.

As prescribed, we start with the normalized function of Eq. (8) . For simplicity but with sufficient generality, let $\psi_{1}(x)$ be a bump function with $\psi_{1}(x)=0$ for $x<0$ or $x>a>0$, and let $\psi_{2}(x)=$ $\psi_{1}(x-D)$, with $D>a$ so that $\psi_{1}(x) \psi_{2}(x)=0$ as required. Because of the generality in choosing $\psi_{1}(x)$, we obtain an unlimited number of M-indeterminate densities $P(r)$ for each operator/class.

\subsection{Example 1}

Consider the class of densities generated by the operator

$$
\mathbf{A}=\frac{1}{i} \frac{d}{d x}
$$

We note that this operator is the momentum operator in the position representation in quantum mechanics (with $\hbar=1$ ) [17, 18]. As previously mentioned, it also appears in the study of cumulants [12, 13] and the Gram-Charlier series [14]. A basic property of the operator is that it shifts a function,

$$
e^{i r \mathbf{A}} f(x)=f(x+r)
$$

Solving the eigenvalue problem for $\mathbf{A}$ yields the eigenfunctions

$$
u(r, x)=\frac{1}{\sqrt{2 \pi}} e^{i r x}
$$

Therefore, we have

$$
\begin{aligned}
F(r) & =\frac{1}{\sqrt{2 \pi}} \int_{-\infty}^{\infty} \psi(x) e^{-i r x} d x \\
& =\frac{1}{\sqrt{2}}\left(F_{1}(r)+e^{i \beta} F_{2}(r)\right) \\
& =\frac{1}{\sqrt{2}} F_{1}(r)\left(1+e^{i(\beta-r D)}\right)
\end{aligned}
$$

where

$$
F_{1}(r)=\frac{1}{\sqrt{2 \pi}} \int_{0}^{a} \psi_{1}(x) e^{-i r x} d x
$$

and

$$
\begin{aligned}
F_{2}(r) & =\frac{1}{\sqrt{2 \pi}} \int_{D}^{a+D} \psi_{2}(x) e^{-i r x} d x \\
& =\frac{1}{\sqrt{2 \pi}} \int_{0}^{a} \psi_{1}(x) e^{-i r(x+D)} d x \\
& =e^{-i r D} F_{1}(r)
\end{aligned}
$$

Accordingly, the probability density associated with $F(r)$ is

$$
P(r)=|F(r)|^{2}=\left|F_{1}(r)\right|^{2}[1+\cos (r D-\beta)]
$$


which, as anticipated from Theorem 1 , depends on $\beta$. However, the moments $\mathrm{E}\left[R^{n}\right]$ are independent of $\beta$ and are given by

$$
\begin{aligned}
\mathrm{E}\left[R^{n}\right]= & \int_{-\infty}^{\infty} r^{n}\left|F_{1}(r)\right|^{2}[1+\cos (r D-\beta)] d r \\
= & \int_{-\infty}^{\infty} \psi^{*}(x) \mathbf{A}^{n} \psi(x) d x \\
= & \frac{1}{2} \int_{-\infty}^{\infty}\left(\psi_{1}^{*}(x)+e^{-i \beta} \psi_{1}^{*}(x-D)\right)\left(\frac{1}{i} \frac{d}{d x}\right)^{n}\left(\psi_{1}(x)+e^{i \beta} \psi_{1}(x-D)\right) d x \\
= & \frac{1}{2} \int_{-\infty}^{\infty} \psi_{1}^{*}(x)\left(\frac{1}{i} \frac{d}{d x}\right)^{n} \psi_{1}(x) d x+\frac{1}{2} \int_{-\infty}^{\infty} \psi_{1}^{*}(x-D)\left(\frac{1}{i} \frac{d}{d x}\right)^{n} \psi_{1}(x-D) d x \\
& +\operatorname{Re}\left(e^{-i \beta} \int_{-\infty}^{\infty} \psi_{1}^{*}(x-D)\left(\frac{1}{i} \frac{d}{d x}\right)^{n} \psi_{1}(x) d x\right)
\end{aligned}
$$

But, since $D>a>0$ and $\psi_{1}(x)=0$ for $x \notin(0, a)$, it follows that

$$
\int_{-\infty}^{\infty} \psi_{1}^{*}(x-D)\left(\frac{1}{i} \frac{d}{d x}\right)^{n} \psi_{1}(x) d x=0
$$

Hence, the moments are

$$
\begin{aligned}
\mathrm{E}\left[R^{n}\right] & =\frac{1}{2} \int_{-\infty}^{\infty} \psi_{1}^{*}(x)\left(\frac{1}{i} \frac{d}{d x}\right)^{n} \psi_{1}(x) d x+\frac{1}{2} \int_{-\infty}^{\infty} \psi_{1}^{*}(x-D)\left(\frac{1}{i} \frac{d}{d x}\right)^{n} \psi_{1}(x-D) d x \\
& =\int_{-\infty}^{\infty} \psi_{1}^{*}(x)\left(\frac{1}{i} \frac{d}{d x}\right)^{n} \psi_{1}(x) d x
\end{aligned}
$$

which do not depend on $\beta$ and therefore all the densities $P(r)$ parameterized by $\beta$ per Eq. (15) are M-indeterminate, as has been shown previously [22, 23]. Note that Eq. (15) is of the form of a Stieltjes class, Eq. (2); however, as the following example shows, other self-adjoint operators can lead to new classes of M-indeterminate densities that can not be expressed in the form of a Stieltjes class.

\subsection{Example 2}

As a second example of a class of M-indeterminate densities, for the operator we take [25]

$$
\mathbf{A}=c x+\frac{1}{i} \frac{d}{d x}
$$

where $c$ is a real number. This operator is the sum of the position plus momentum operators, properly dimensionalized. A property of this operator is that

$$
e^{i r \mathbf{A}} f(x)=e^{i c r x-i c r^{2} / 2} f(x-r)
$$

The eigenfunctions are

$$
u(r, x)=\frac{1}{\sqrt{2 \pi}} e^{i\left(r x-c x^{2} / 2\right)}
$$

Hence, the $r$-transform here is given by [25]

$$
F(r)=\frac{1}{\sqrt{2 \pi}} \int_{-\infty}^{\infty} \psi(x) e^{-i r x+i c x^{2} / 2} d x
$$


For $\psi(x)$ as defined by Eq. (8), we thus have

$$
F(r)=\frac{1}{\sqrt{2}}\left(F_{1}(r)+e^{i \beta} F_{2}(r)\right)
$$

where

$$
F_{1}(r)=\frac{1}{\sqrt{2 \pi}} \int_{0}^{a} e^{-i r x+i c x^{2} / 2} \psi_{1}(x) d x
$$

and

$$
\begin{aligned}
F_{2}(r) & =\frac{1}{\sqrt{2 \pi}} \int_{D}^{D+a} e^{-i r x+i c x^{2} / 2} \psi_{2}(x) d x=\frac{1}{\sqrt{2 \pi}} \int_{D}^{D+a} e^{-i r x+i c x^{2} / 2} \psi_{1}(x-D) d x \\
& =\frac{1}{\sqrt{2 \pi}} e^{-i r D+i c D^{2} / 2} \int_{0}^{a} e^{-i(r-c D) x+i c x^{2} / 2} \psi_{1}(x) d x \\
& =e^{-i r D+i c D^{2} / 2} F_{1}(r-c D)
\end{aligned}
$$

Hence,

$$
F(r)=\frac{1}{\sqrt{2}}\left[F_{1}(r)+e^{i \beta} e^{-i r D+i c D^{2} / 2} F_{1}(r-c D)\right]
$$

and the probability density associated with $F(r)$ is

$$
\begin{aligned}
P(r) & =|F(r)|^{2} \\
& =\frac{1}{2}\left[\left|F_{1}(r)\right|^{2}+\left|F_{1}(r-c D)\right|^{2}+2 \operatorname{Re}\left\{F_{1}^{*}(r) e^{i \beta} e^{-i r D+i c D^{2} / 2} F_{1}(r-c D)\right\}\right]
\end{aligned}
$$

Here again, while the densities $P(r)$ depend on $\beta$, the moments do not by virtue of Eq. (13), and hence these densities are M-indeterminate. Note that this case reduces to the previous case, Eq. (15), for $c=0$. However, for $c \neq 0$, we can not express these M-indeterminate densities in the form of Eq. (2).

\subsection{Example 3}

The operator

$$
\mathbf{A}=\frac{1}{2 i}\left(x \frac{d}{d x}+\frac{d}{d x} x\right)=\frac{1}{i}\left(x \frac{d}{d x}+\frac{1}{2}\right)=\frac{1}{i}\left(\frac{d}{d x} x-\frac{1}{2}\right)
$$

arises in the compression or dilation of functions. In particular, [26]

$$
e^{i r \mathbf{A}} f(x)=e^{r / 2} f\left(e^{r} x\right)
$$

The compression/dilation factor is $e^{r}$; for $0<e^{r}<1$, the function $f(x)$ is dilated, while for $e^{r}>1$, the function is compressed. The factor $e^{r / 2}$ preserves normalization of $f(x)$ :

$$
\int_{-\infty}^{\infty}|f(x)|^{2} d x=\int_{-\infty}^{\infty}\left|e^{r / 2} f\left(e^{r} x\right)\right|^{2} d x=1
$$

Solving the eigenvalue problem for $\mathbf{A}$ yields the eigenfunctions

$$
u(r, x)=\frac{1}{\sqrt{2 \pi}} \frac{e^{i r \ln x}}{\sqrt{x}}, \quad x \geq 0
$$


Hence, for one sided $\psi(x)$ we have

$$
F(r)=\int_{0}^{\infty} \psi(x) u^{*}(r, x) d x=\frac{1}{\sqrt{2 \pi}} \int_{0}^{\infty} \frac{e^{-i r \ln x}}{\sqrt{x}} \psi(x) d x
$$

which we note is a Mellin transform with argument $-i r+1 / 2$.

Now for our problem,

$$
F(r)=\frac{1}{\sqrt{2}}\left(F_{1}(r)+e^{i \beta} F_{2}(r)\right)
$$

where

$$
F_{1}(r)=\frac{1}{\sqrt{2 \pi}} \int_{0}^{a} \frac{e^{-i r \ln x}}{\sqrt{x}} \psi_{1}(x) d x
$$

and

$$
\begin{aligned}
F_{2}(r) & =\frac{1}{\sqrt{2 \pi}} \int_{D}^{D+a} \psi_{1}(x-D) \frac{e^{-i r \ln x}}{\sqrt{x}} d x \\
& =\frac{1}{\sqrt{2 \pi}} \int_{0}^{a} \psi_{1}(x) \frac{e^{-i r \ln (x+D)}}{\sqrt{x+D}} d x
\end{aligned}
$$

Therefore

$$
\begin{aligned}
F(r) & =\frac{1}{\sqrt{2}}\left(\frac{1}{\sqrt{2 \pi}} \int_{0}^{a} \frac{e^{-i r \ln x}}{\sqrt{x}} \psi_{1}(x) d x+e^{i \beta} \frac{1}{\sqrt{2 \pi}} \int_{0}^{a} \psi_{1}(x) \frac{e^{-i r \ln (x+D)}}{\sqrt{x+D}} d x\right) \\
& =\frac{1}{\sqrt{2}} \frac{1}{\sqrt{2 \pi}} \int_{0}^{a}\left(\frac{e^{-i r \ln x}}{\sqrt{x}}+e^{i \beta} \frac{e^{-i r \ln (x+D)}}{\sqrt{x+D}}\right) \psi_{1}(x) d x
\end{aligned}
$$

The probability densities associated with $F(r)$ are given by

$$
P(r)=|F(r)|^{2}=\frac{1}{4 \pi}\left|\int_{0}^{a}\left(\frac{e^{-i r \ln x}}{\sqrt{x}}+e^{i \beta} \frac{e^{-i r \ln (x+D)}}{\sqrt{x+D}}\right) \psi_{1}(x) d x\right|^{2}
$$

As before, the densities depend on $\beta$, however the moments do not which is straightforward to show via the operator approach of Eq. (7).

It is of interest to contrast the operator approach with the calculation of the moments in the usual way, namely

$$
\begin{aligned}
\mathrm{E}\left[R^{n}\right] & =\int_{-\infty}^{\infty} r^{n}|F(r)|^{2} d r=\frac{1}{4 \pi} \int_{-\infty}^{\infty} r^{n}\left|\int_{0}^{a}\left(\frac{e^{-i r \ln x}}{\sqrt{x}}+e^{i \beta} \frac{e^{-i r \ln (x+D)}}{\sqrt{x+D}}\right) \psi_{1}(x) d x\right|^{2} d r \\
& =\frac{1}{4 \pi} \int_{-\infty}^{\infty} r^{n} \int_{0}^{a} \int_{0}^{a}\left(\frac{e^{-i r \ln x}}{\sqrt{x}}+e^{i \beta} \frac{e^{-i r \ln (x+D)}}{\sqrt{x+D}}\right)\left(\frac{e^{i r \ln x^{\prime}}}{\sqrt{x^{\prime}}}+e^{-i \beta} \frac{e^{i r \ln \left(x^{\prime}+D\right)}}{\sqrt{x^{\prime}+D}}\right) \psi_{1}^{*}\left(x^{\prime}\right) \psi_{1}(x) d x d x^{\prime} d r
\end{aligned}
$$

Upon expanding we obtain

$$
\begin{aligned}
\mathrm{E}\left[R^{n}\right] & =\frac{1}{4 \pi} \int_{-\infty}^{\infty} r^{n}\left|\int_{0}^{a} \frac{e^{-i r \ln x}}{\sqrt{x}} \psi_{1}(x) d x\right|^{2} d r+\frac{1}{4 \pi} \int_{-\infty}^{\infty} r^{n} \int_{0}^{a}\left|\int_{0}^{a} \frac{e^{-i r \ln (x+D)}}{\sqrt{x+D}} \psi_{1}(x) d x\right| d r \\
& +\frac{1}{4 \pi} \int_{-\infty}^{\infty} r^{n} \int_{0}^{a} \int_{0}^{a}\left(e^{-i \beta} \frac{e^{-i r \ln x}}{\sqrt{x}} \frac{e^{i r \ln \left(x^{\prime}+D\right)}}{\sqrt{x^{\prime}+D}}+e^{i \beta} \frac{e^{i r \ln x^{\prime}}}{\sqrt{x^{\prime}}} \frac{e^{-i r \ln (x+D)}}{\sqrt{x+D}}\right) \psi_{1}^{*}\left(x^{\prime}\right) \psi_{1}(x) d x d x^{\prime} d r
\end{aligned}
$$


The first two terms are independent of $\beta$. Therefore, to show that the moments are independent of $\beta$, we need to show that

$$
\int_{-\infty}^{\infty} \int_{0}^{a} \int_{0}^{a} r^{n}\left(e^{-i \beta} \frac{e^{-i r \ln x}}{\sqrt{x}} \frac{e^{i r \ln \left(x^{\prime}+D\right)}}{\sqrt{x^{\prime}+D}}+e^{i \beta} \frac{e^{i r \ln x^{\prime}}}{\sqrt{x^{\prime}}} \frac{e^{-i r \ln (x+D)}}{\sqrt{x+D}}\right) \psi_{1}^{*}\left(x^{\prime}\right) \psi_{1}(x) d x d x^{\prime} d r=0
$$

for $D>a>0$ and $\psi_{1}(x)=0, x \notin(0, a)$.

Consider

$$
\begin{aligned}
I & =\int_{-\infty}^{\infty} \int_{0}^{a} \int_{0}^{a} r^{n}\left(\frac{e^{-i r \ln x}}{\sqrt{x}} \frac{e^{i r \ln \left(x^{\prime}+D\right)}}{\sqrt{x^{\prime}+D}}\right) \psi_{1}^{*}\left(x^{\prime}\right) \psi_{1}(x) d x d x^{\prime} d r \\
& =\int_{-\infty}^{\infty} \int_{0}^{a} \int_{D}^{D+a} r^{n} \frac{e^{i r \ln \left(x^{\prime} / x\right)}}{\sqrt{x x^{\prime}}} \psi_{1}^{*}\left(x^{\prime}-D\right) \psi_{1}(x) d x^{\prime} d x d r
\end{aligned}
$$

Making the variable substitution $y=\ln \left(x^{\prime} / x\right)$ with $d x^{\prime}=x e^{y} d y$ we obtain

$$
\begin{aligned}
I & =\int_{0}^{a} \int_{\ln (D / x)}^{\ln ((D+a) / x)} \int_{-\infty}^{\infty} r^{n} \frac{e^{i r y}}{\sqrt{x^{2} e^{y}}} \psi_{1}^{*}\left(x e^{y}-D\right) \psi_{1}(x) x e^{y} d r d y d x \\
& =\int_{0}^{a} \int_{\ln (D / x)}^{\ln ((D+a) / x)} \int_{-\infty}^{\infty} r^{n} e^{i r y} \psi_{1}^{*}\left(x e^{y}-D\right) \psi_{1}(x) e^{y / 2} d r d y d x \\
& =2 \pi \int_{0}^{a} \int_{\ln (D / x)}^{\ln ((D+a) / x)}\left\{\left(\frac{1}{i} \frac{d}{d y}\right)^{n} \delta(y)\right\} \psi_{1}^{*}\left(x e^{y}-D\right) e^{y / 2} \psi_{1}(x) d y d x \\
& =\left.2 \pi \int_{0}^{a} i^{n}\left\{\left(\frac{d}{d y}\right)^{n} \psi_{1}^{*}\left(x e^{y}-D\right) e^{y / 2}\right\}\right|_{y=0} \psi_{1}(x) d x
\end{aligned}
$$

Evaluation leads to

$$
I=2 \pi \int_{0}^{a} \sum_{k=0}^{n} \frac{i^{n} x^{k}}{2^{n-k}}\left(\begin{array}{l}
n \\
k
\end{array}\right) \psi_{1}^{*(k)}(x-D) \psi_{1}(x) d x=0
$$

where the last step follows since $\psi_{1}^{*(k)}(x-D) \psi_{1}(x)=0$ for $D>a$. By an identical derivation, we obtain

$$
\int_{-\infty}^{\infty} \int_{0}^{a} \int_{0}^{a} r^{n}\left(\frac{e^{i r \ln x^{\prime}}}{\sqrt{x^{\prime}}} \frac{e^{-i r \ln (x+D)}}{\sqrt{x+D}}\right) \psi_{1}^{*}\left(x^{\prime}\right) \psi_{1}(x) d x d x^{\prime} d r=0
$$

for $D>a$. Hence Eq. (20) holds, and the moments are independent of $\beta$, as previously determined via the operator approach.

\subsection{Example 4}

For the operator we take

$$
\mathbf{A}=-\frac{d^{2}}{d x^{2}}-\frac{1}{c} x
$$

where $c$ is a constant. This case is motivated by what is called the constant force Hamiltonian in quantum mechanics. It consists of the kinetic energy operator, $-\frac{d^{2}}{d x^{2}}$ (with $\hbar=1$ and mass equal to $1 / 2$ ), plus the potential energy term, $-\frac{1}{c} x$, which corresponds to a constant force in the positive $x$ direction with magnitude $1 / c$. 
The eigenvalue problem is

$$
\left(-\frac{d^{2}}{d x^{2}}-\frac{1}{c} x\right) u(x, r)=r u(x, r)
$$

where $r$ are the eigenvalues. The eigenvalue problem can be solved in any representation. To solve this eigenvalue problem, it is simpler to convert to the dual Fourier domain by defining

$$
v(p, r)=\frac{1}{\sqrt{2 \pi}} \int_{-\infty}^{\infty} e^{-i p x} u(x, r) d x
$$

in which case the eigenvalue problem becomes

$$
\left(p^{2}-\frac{i}{c} \frac{d}{d p}\right) v(p, r)=r v(p, r)
$$

The eigenfunctions, $v(p, r)$, are

$$
v(p, r)=\sqrt{\frac{c}{2 \pi}} e^{i c\left(r p-p^{3} / 3\right)}
$$

and the corresponding $u(x, r)$ are then given by

$$
\begin{aligned}
u(x, r) & =\frac{1}{\sqrt{2 \pi}} \int_{-\infty}^{\infty} e^{-i p x} v(p, r) d p \\
& =\sqrt{\frac{c}{2 \pi}} \int_{-\infty}^{\infty} e^{-i p x} e^{i c\left(r p-p^{3} / 3\right)} d p
\end{aligned}
$$

These eigenfunctions can be expressed in terms of Airy functions but that is not necessary for our considerations.

As with $\psi(x)$, we can expand $\varphi(p)$ in terms of the eigenfunctions via

$$
\varphi(p)=\sqrt{\frac{c}{2 \pi}} \int_{-\infty}^{\infty} F(r) e^{i c\left(r p-p^{3} / 3\right)} d r
$$

where

$$
F(r)=\sqrt{\frac{c}{2 \pi}} \int_{-\infty}^{\infty} \varphi(p) e^{-i c\left(r p-p^{3} / 3\right)} d p
$$

Now for our problem, let $\varphi(p)$ consist of two functions with disjoint support,

$$
\varphi(p)=\frac{1}{\sqrt{2}}\left(\varphi_{1}(p)+e^{i \beta} \varphi_{2}(p)\right)
$$

where $\varphi_{1}(p)=0$ for $p<0$ or $p>a>0$, and $\varphi_{2}(p)=\varphi_{1}(p-D)$, with $D>a$ so that $\varphi_{1}(p) \varphi_{2}(p)=0$. Then, it follows that

$$
F(r)=\frac{1}{\sqrt{2}}\left(F_{1}(r)+e^{i \beta} F_{2}(r)\right)
$$

where

$$
\begin{aligned}
F_{1}(r) & =\sqrt{\frac{c}{2 \pi}} \int_{0}^{a} \varphi_{1}(p) e^{i c\left(r p-p^{3} / 3\right)} d p \\
F_{2}(r) & =\sqrt{\frac{c}{2 \pi}} \int_{D}^{D+a} \varphi_{2}(p) e^{i c\left(r p-p^{3} / 3\right)} d p \\
& =\sqrt{\frac{c}{2 \pi}} \int_{0}^{a} \varphi_{2}(p+D) e^{i c\left(r(p+D)-(p+D)^{3} / 3\right)} d p
\end{aligned}
$$


The densities associated with $F(r)$ are

$$
P(r)=|F(r)|^{2}=\frac{1}{2}\left(\left|F_{1}(r)\right|^{2}+\left|F_{2}(r)\right|^{2}+2 \operatorname{Re}\left\{F_{1}^{*}(r) e^{i \beta} F_{2}(r)\right\}\right)
$$

which clearly depend on $\beta$ (even though $\varphi_{1}(p) \varphi_{2}(p)=0$, in general $F_{1}(r) F_{2}(r) \neq 0$ ).

The moments $\mathrm{E}\left[R^{n}\right]$ are given by

$$
\mathrm{E}\left[R^{n}\right]=\int_{-\infty}^{\infty} r^{n} P(r) d r=\frac{1}{2} \int_{-\infty}^{\infty} r^{n}\left(\left|F_{1}(r)\right|^{2}+\left|F_{2}(r)\right|^{2}+2 \operatorname{Re}\left\{F_{1}^{*}(r) e^{i \beta} F_{2}(r)\right\}\right) d r
$$

The first two terms are clearly independent of $\beta$; however, so is the last term, by virtue of the fact that

$$
\int_{-\infty}^{\infty} F_{1}^{*}(r) r^{n} F_{2}(r) d r=\int_{-\infty}^{\infty} \varphi_{1}^{*}(p) \mathbf{A}^{n} \varphi_{2}(p) d p=0
$$

which follows from the fact that $\varphi_{1}(p)$ and $\varphi_{2}(p)$ are non-overlapping and the support of $\mathbf{A}^{n} \varphi_{2}(p)$ is equal to the support of $\varphi_{2}(p)$. Accordingly, the densities $P(r)$ (Eq. (22) ) are M-indeterminate since they depend on the parameter $\beta$ but the moments do not.

\section{Discrete Case}

The generation of M-indeterminate densities that are discrete follows readily from the previous considerations, which we succinctly present here. In this section, $R$ is a real discrete random variable that assumes possible values $r_{1}, r_{2}, r_{3} \ldots$. with corresponding probabilities $P\left(r_{n}\right)$.

For the case of self-adjoint operators $\mathbf{A}$ that give discrete eigenvalues, we write the eigenvalue problem as

$$
\mathbf{A} u_{n}(x)=r_{n} u_{n}(x)
$$

The eigenfunctions $u_{n}(x)$ are complete and orthogonal, hence, any continuous function in $L^{2}$ can be expanded as

$$
\psi(x)=\sum_{n} c_{n} u_{n}(x)
$$

where the coefficients $c_{n}$ are given by

$$
c_{n}=\int_{-\infty}^{\infty} \psi(x) u_{n}^{*}(x) d x
$$

and the normalization is such that

$$
\int_{-\infty}^{\infty}|\psi(x)|^{2} d x=\sum_{n}\left|c_{n}\right|^{2}=1
$$

The eigenvalues are the discrete random variables and their associated probabilities are given by

$$
P\left(r_{n}\right)=\left|c_{n}\right|^{2}=\left|\int_{-\infty}^{\infty} \psi(x) u_{n}^{*}(x) d x\right|^{2}
$$

with expected value

$$
\mathrm{E}[R]=\sum_{n} r_{n}\left|c_{n}\right|^{2}
$$


By Lemma 1, we have equivalently that

$$
\mathrm{E}[R]=\int_{-\infty}^{\infty} \psi^{*}(x) \mathbf{A} \psi(x) d x
$$

and more generally,

$$
\mathrm{E}[g(R)]=\sum_{n} g\left(r_{n}\right)\left|c_{n}\right|^{2}=\int_{-\infty}^{\infty} \psi^{*}(x) g(\mathbf{A}) \psi(x) d x
$$

Theorem 2 The discrete probability density functions given by Eq. (23) with $\psi(x)$ as defined in Eq. (8) are $M$-indeterminate for self-adjoint operators for which the support of $\mathbf{A}^{n} \psi(x)$ equals the support of $\psi(x)$.

Proof. The proof mirrors that of Theorem 1. Briefly, we have

$$
\begin{aligned}
P\left(r_{n}\right) & =\left|\int_{-\infty}^{\infty} \psi(x) u_{n}^{*}(x) d x\right|^{2}=\frac{1}{2} \int_{-\infty}^{\infty} \int_{-\infty}^{\infty}\left(\psi_{1}^{*}\left(x^{\prime}\right)+e^{-i \beta} \psi_{2}^{*}\left(x^{\prime}\right)\right) u_{n}\left(x^{\prime}\right)\left(\psi_{1}(x)+e^{i \beta} \psi_{2}(x)\right) u_{n}^{*}(x) d x d x^{\prime} \\
& =\frac{1}{2}\left(P_{1}\left(r_{n}\right)+P_{2}\left(r_{n}\right)\right)+\frac{1}{2}\left(e^{i \beta} c_{n}^{*(1)} c_{n}^{(2)}+e^{-i \beta} c_{n}^{*(2)} c_{n}^{(1)}\right)
\end{aligned}
$$

where

$$
\begin{aligned}
& P_{1}\left(r_{n}\right)=\left|c_{n}^{(1)}\right|^{2} \quad ; \quad P_{2}\left(r_{n}\right)=\left|c_{n}^{(2)}\right|^{2} \\
& c_{n}^{(1)}=\int_{-\infty}^{\infty} \psi_{1}(x) u_{n}^{*}(x) d x ; \quad c_{n}^{(2)}=\int_{-\infty}^{\infty} \psi_{2}(x) u_{n}^{*}(x) d x
\end{aligned}
$$

In general the product $c_{n}^{*(1)} c_{n}^{(2)}$ is not identically zero, in which case $P\left(r_{n}\right)$ will depend on $\beta$. On the other hand, as in the continuous case, because $\psi_{1}(x)$ and $\psi_{2}(x)$ have disjoint support, the moments are independent of $\beta$,

$$
\begin{aligned}
\mathrm{E}\left[R^{n}\right]=\sum_{n=0}^{\infty} r^{n}\left|c_{n}\right|^{2} & =\sum_{n=0}^{\infty} r^{n}\left|\frac{1}{\sqrt{2}}\left(c_{n}^{(1)}+e^{i \beta} c_{n}^{(2)}\right)\right|^{2} \\
& =\frac{1}{2} \int_{-\infty}^{\infty}\left(\psi_{1}^{*}(x)+e^{-i \beta} \psi_{2}^{*}(x)\right) \mathbf{A}^{n}\left(\psi_{1}(x)+e^{i \beta} \psi_{2}(x)\right) d x \\
& =\frac{1}{2} \mathrm{E}\left[R^{n}\right]_{1}+\frac{1}{2} \mathrm{E}\left[R^{n}\right]_{2}+e^{-i \beta} \int_{-\infty}^{\infty} \psi_{2}^{*}(x) \mathbf{A}^{n} \psi_{1}(x) d x+e^{i \beta} \int_{-\infty}^{\infty} \psi_{1}^{*}(x) \mathbf{A}^{n} \psi_{2}(x) d x
\end{aligned}
$$

where $\mathrm{E}\left[R^{n}\right]_{1}$ and $\mathrm{E}\left[R^{n}\right]_{2}$ are the expectation values

$$
\begin{aligned}
\mathrm{E}\left[R^{n}\right]_{1} & =\sum_{n=0}^{\infty} r^{n}\left|c_{n}^{(1)}\right|^{2}=\int_{-\infty}^{\infty} \psi_{1}^{*}(x) \mathbf{A}^{n} \psi_{1}(x) d x \\
\mathrm{E}\left[R^{n}\right]_{2} & =\sum_{n=0}^{\infty} r^{n}\left|c_{n}^{(2)}\right|^{2}=\int_{-\infty}^{\infty} \psi_{2}^{*}(x) \mathbf{A}^{n} \psi_{2}(x) d x
\end{aligned}
$$

But since $\psi_{1}(x) \psi_{2}(x)=0$, Eq. (13) holds, by which it follows that the moments are independent of $\beta$ and therefore all the (different) $P\left(r_{n}\right)$ given by Eq. (26) have the same moments. 


\section{Conclusion}

M-indeterminate densities are those that are not uniquely determined by their moments. Constructing such densities and/or determining whether or not a density is M-determinate has historically been a challenging problem, although many such densities and tests have been discovered since the issue was first considered by Stieltjes.

From a quantum perspective, the issue first arose with the consideration of two non-overlapping wave functions in position space as given by Eq. (8). Aharonov et al. showed that the moments of both the position density and of the momentum density are independent of the parameter $\beta$ [20, 21, 22]. This latter result renders the momentum density M-indeterminate, since the density itself does depend on $\beta$ [22]. The analogous case of non-overlapping momentum wave functions has also been considered [27].

We have shown that the unique way in which probability densities are obtained in quantum mechanics gives rise to a simple procedure for constructing classes of M-indeterminate densities. Namely, we start with a complete and orthogonal set of functions $u(r, x)$ that are solutions of the eigenvalue problem for a self-adjoint operator $\mathbf{A}$ with eigenvalues $r$,

$$
\mathbf{A} u(r, x)=r u(r, x)
$$

Then for the generally complex, continous function $\psi(x)$ one forms the transform

$$
F(r)=\int_{-\infty}^{\infty} \psi(x) u^{*}(r, x) d x
$$

Normalizing such that $\int_{-\infty}^{\infty}|\psi(x)|^{2} d x=1$, it follows that $P(r)=|F(r)|^{2}$ is a proper probability density.

Different classes of densities $P(r)$ are obtained by choosing different operators and/or functions $\psi(x)$. The densities in each class will be M-indeterminate when the following conditions hold:

1. The function $\psi(x)$ is of the form $\psi(x)=\frac{1}{\sqrt{2}}\left(\psi_{1}(x)+e^{i \beta} \psi_{2}(x)\right)$, where $\psi_{1}(x)$ and $\psi_{2}(x)$ are each normalized to one and, crucially, have disjoint support:

$$
\psi_{1}(x) \psi_{2}(x)=0
$$

2. It follows that the function in the $r$-domain is $F(r)=\int_{-\infty}^{\infty} \psi(x) u^{*}(r, x) d x=\frac{1}{\sqrt{2}}\left(F_{1}(r)+e^{i \beta} F_{2}(r)\right)$. However, while the functions $\psi_{1}, \psi_{2}$ do not overlap in the $x$-domain, they do overlap in the $r$ domain:

$$
F_{1}(r) F_{2}(r) \neq 0
$$

3. For any nonnegative integer $n, \mathbf{A}^{n} \psi_{k}(x)$ has the same support in $x$ as does $\psi_{k}(x)$. Together with condition 1 above, it therefore follows that

$$
\psi_{1}^{*}(x) \mathbf{A}^{n} \psi_{2}(x)=\psi_{2}^{*}(x) \mathbf{A}^{n} \psi_{1}(x)=0
$$

This will be the case for (finite order) differential operators, and many other operators as shown in sec. 4. 
Condition 2 means that the densities $P(r)$ will depend on the parameter, $\beta$. However, Conditions 1 and 3 render the moments $\mathrm{E}\left[R^{n}\right]$ independent of $\beta$, which follows readily from the operator-procedure for calculating moments, namely Eq. (7), which we re-state specifically in terms of moments,

$$
\mathrm{E}\left[R^{n}\right]=\int_{-\infty}^{\infty} r^{n}|F(r)|^{2} d r=\int_{-\infty}^{\infty} \psi^{*}(x) \mathbf{A}^{n} \psi(x) d x
$$

One can also readily show that

$$
\int_{-\infty}^{\infty} F_{1}^{*}(r) r^{n} F_{2}(r) d r=\int_{-\infty}^{\infty} \psi_{1}^{*}(x) \mathbf{A}^{n} \psi_{2}(x) d x
$$

by which it becomes clear, via Condition 3, that the moments are independent of $\beta$. Hence, the densites $P(r)$ are M-indeterminate.

\section{$7 \quad$ Acknowledgments}

R. Sala Mayato acknowledges funding by the Spanish MINECO and FEDER, grant FIS2017-82855-P (MINE CO/ FEDER, UE), and by M. Payne.

\section{References}

[1] Feller, W.: An Introduction to Probability Theory and Its Applications, Vol. 2. John Wiley and Sons, New York (1966)

[2] Stoyanov, J.: Counterexamples in Probability, 3rd ed. Dover (2013)

[3] Heyde, C.C.: On a Property of the Lognormal Distribution. Journal of the Royal Statistical Society: Series B, 25, 392, 1963.

[4] Akhiezer, N.I.: The Classical Moment Problem and Some Related Questions in Analysis. Hafner Publishing Co., New York (1965)

[5] Shohat, J.A., Tamarkin, J.D.: The Problem of Moments. Mathematical Surveys, vol. I. American Mathematical Society, New York (1943)

[6] Stoyanov, J.: Stieltjes classes for moment-indeterminate probability distributions. J. Applied Probab. 41, 281-294 (2004)

[7] Stoyanov, J.: Determinacy of distributions by their moments. Intl. Conf. Math. Statist. Modeling in Honor of Enrique Castillo. (2006)

[8] Stoyanov, J., Tolmatz, L.: Method for constructing Stieltjes classes for m-indeterminate probability distributions. Appl. Math. and Comp. 165, 669-685 (2005)

[9] Gut, A.: On the moment problem. Bernoulli 8, 407-421 (2002) 
[10] Kleiber, C.: The generalized lognormal distribution and the Stieltjes moment problem. J. Theor. Probab. 27, 1167-1177 (2014)

[11] Schmüdgen, K.: The Moment Problem. Springer International Publishing AG, Cham, Switzerland (2017)

[12] Stuart, A., Ord, K. K.: Kendall's Advanced Theory of Statistics. Halsted Press, NY (1994)

[13] Hald, A.: The early history of the cumulants and the Gram-Charlier series. Internat. Stat. Rev. 68(2), 137-153 (2000)

[14] Cohen, L.: Generalization of the Edgeworth and Gram-Charlier series and quasi-probability densities. Research Inst. for Math. Sciences. Kyoto University. 2056, 1-33 (2017)

[15] Cohen, L.: Are there quantum operators and wave functions in standard probability theory? In: Wong, M. W., Zhu, H. (eds.) Pseudo-Differential Operators: Groups, Geometry and Applications, pp. 133-147. Birkhäuser Mathematics (2017)

[16] Cohen, L.: Rules of probability in quantum mechanics. Found. Phys. 18(10), 983-998 (1988)

[17] Bohm, D: Quantum Theory. Prentice-Hall, New York, 1951.

[18] Merzbacher, E.: Quantum Mechancs. John Wiley \& Sons, Inc.,1998.

[19] Morse, P. and Feshbach, H.: Methods of Theoretical Physics, Part I. McGraw-Hill Book Company, New York, 1953.

[20] Aharonov, Y., Pendleton, H., Petersen, A.: Modular variables in quantum theory. Int. J. Theor. Phys. 2(3), 213-230 (1969)

[21] Aharonov, Y., Rohrlich, D.: Quantum Paradoxes. Wiley-VCH, Weinheim, Germany (2005)

[22] Semon, M.D., Taylor, J.R.: Expectation values in the Aharonov-Bohm effect. Nuovo Cim. 97, 25-40 (1987)

[23] Sala Mayato, R, Loughlin, P., Cohen, L.: M-indeterminate distributions in quantum mechanics and the non-overlapping wave function paradox. Physics Letters A 382, 2914-2921 (2018)

[24] Tu, L. W.: An Introduction to Manifolds, Second Edition. Springer, NY (2011)

[25] Cohen, L.: The Weyl Operator and its Generalization. Springer, Basel (2013)

[26] Cohen, L.: The scale representation. IEEE Trans. Signal Process. 41, 3275-3292 (1993)

[27] Wang, D-W., Scully, M.O.: Heisenberg limit superradiant superresolving metrology. Phys. Rev. Letters 113, 083601 (2014) 